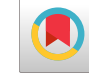

\title{
A Fatal Case of Bloodstream Infection by Fusarium Solani in a Patient with Adrenocortical Carcinoma From Isfahan, Iran
}

\author{
Alireza Rajabzadeh ${ }^{1}$, Dariush Shokri ${ }^{2}$, Shima Aboutalebian ${ }^{3}$, Hamid Morovati ${ }^{4}$, Abdolrasoul \\ Mohammadi ${ }^{3}$, Reza Eshraghi Samani ${ }^{5}$ and Rasoul Mohammadi $\mathbb{1}^{6, *}$ \\ ${ }^{1}$ Infectious Diseases Specialist, Isfahan Milad Hospital, Isfahan, Iran \\ ${ }^{2}$ Department of Microbiology, Faculty of Biological Sciences and Technology, Shahid Ashrafi Esfahani University, Isfahan, Iran \\ ${ }^{3}$ Department of Medical Parasitology and Mycology, School of Medicine, Isfahan University of Medical Sciences, Isfahan, Iran \\ ${ }^{4}$ Department of Medical Parasitology and Mycology, School of Medicine, Shiraz University of Medical Sciences, Shiraz, Iran \\ ${ }^{5}$ Department of Surgery, Isfahan University of Medical Sciences, Isfahan, Iran \\ ${ }^{6}$ Department of Medical Parasitology and Mycology, School of Medicine, Infectious Diseases and Tropical Medicine Research Center, Isfahan University of Medical Sciences, \\ Isfahan, Iran \\ "Corresponding author: Department of Medical Parasitology and Mycology, School of Medicine, Infectious Diseases and Tropical Medicine Research Center, Isfahan University \\ of Medical Sciences, Isfahan, Iran. Email: dr.rasoul_mohammadi@yahoo.com
}

Received 2019 October 04; Revised 2019 December 07; Accepted 2020 January 11.

\begin{abstract}
Introduction: Fusarium species are hyaline saprophytic fungi that are frequently found in the soil, air, and water. They can cause severe systemic infections in immunocompromised patients. Clinical manifestations depend on the way of entry of the mold and host immune system status. The main ways of entrance are the airways, skin, and mucosal membranes. Disseminated fusariosis often occurs in patients with hematological disorders, patients with cancer, and solid organ transplant recipients.

Case Presentation: Herein we report a case of Fusarium fungemia in a patient with adrenocortical carcinoma from Isfahan, Iran. The patient was a 41-year-old female with stage III adrenal cortical carcinoma. Despite antifungal therapy with liposomal amphotericin $\mathrm{B}$, the patient passed away 6 days after admission. Internal transcribed spacer region sequencing applied for species identification and its sequence deposited in the GenBank (accession number: MK880379).

Conclusions: Since the ideal strategies against invasive fungal infections remain uncertain and the mortality rate is high, we recommend primary prophylaxis with a broad-spectrum antifungal agent for vulnerable patients particularly those admitted to high-risk units such as oncology, hematology, and transplant units.
\end{abstract}

Keywords: Fusarium solani, Fungemia, Adrenocortical Carcinoma, ITS-Sequensing, Isfahan

\section{Introduction}

Invasive fungal infections are on the rise and Fusarium species are important opportunistic filamentous fungi that cause a broad-spectrum of infections such as onychomycosis, fungal keratitis, superficial skin lesions, endophthalmitis, peritonitis, arthritis, and disseminated infections particularly in immunosuppressed patients (1). The clinical forms of Fusarium infections mainly depend on the way of entry for the fungus and host immune system status (2). Persistent fever and skin lesions are the main symptoms despite antibacterial therapy (3). Recent studies have shown that Fusarium species, mostly cause fungemia in immunosuppressed patients (4-6). Limited infections occur in both immunocompetent and immunocompromised hosts, however disseminated fusariosis regularly occur in patients with hematological disorders, cancer pa- tients, and the solid organ transplant recipients (7). In the present study, we reported a case of fungemia wich caused by Fusarium solani; a rare filamentous fungi, in a patient with metastatic cancer from Isfahan, Iran.

\section{Case Presentation}

A 41-year-old female with stage III adrenal cortical carcinoma referred to the Seyed-al-Shohada Hospital, Isfahan University of Medical Sciences, Isfahan, Iran, in February 2018. She had cancer since December 2014 and underwent adrenalectomy and nephrectomy. Cancer chemotherapy was started with lysodren (mitotane) (4 g/day) and streptozotocin (STZ) $\left(500 \mathrm{mg} / \mathrm{m}^{2} /\right.$ day by intravenous injection). After 16 months, metastatic lung tumors and involvement of abdominal lymph nodes were detected in 2016. After surgery, mitotane ( $4 \mathrm{~g} /$ day) was added to her regimen 
for 6 months. After 2 years, on 25-Feb-2018, the patient readmitted to the Department of Oncology in Seyed-alShohada Hospital due to febrile neutropenia (neutrophil count; $430 / \mu \mathrm{L}$, temp; $\left.39.5^{\circ} \mathrm{C}\right)$, malaise, and weight loss (3 $\mathrm{kg})$. Gentamicin $(3 \times 80 \mathrm{mg} \mathrm{IV} /$ day $)$ and vancomycin $(2$ $\times 750 \mathrm{mg}$ IV/day) were started for probable bacteremia and blood culture was requested for her. After two days, she still had fever and got worse. On the third day, liposomal amphotericin B ( $5 \mathrm{mg} / \mathrm{kg} /$ day) was applied for suspected systemic fungal infection and two blood samples were taken on days 2 and 3 . There was no lesion in the patient's body and C-reactive protein (CRP) increased to 279 $\mathrm{mg} / \mathrm{L}$. The patient's consciousness reduced and she passed away 6 days after admission. Five days after death, Fusarium spp. recovered from both blood samples (Figure 1).

For molecular identification, DNA extraction was performed by phenol-chloroform method (8) and ITS1-5. The 8S-ITS2 region was amplified using ITS1 (5'-TCC GTA GGT GAA CCT GCG G-3') and ITS4 (5'-TCC TCC GCT TAT TGA TAT GC-3') primers, and the amplicon was purified and sent for sequencing in forward direction (Bioneer, Korea). The sequencing product was analyzed using Chromas software version 2.3 (http://chromas.software.informer.com/2.4/) and with search in the basic local alignment search tool (BLAST) database at the National Center for Biotechnology (NCBI) about fungal sequences existing in DNA databases (http://blast.ncbi.nlm.nih.gov/Blast.cgi).

After molecular analysis, Fusarium solani was determined as the etiological agent of fungemia in above mentioned case and its sequence deposited in the GenBank (accession number: MK880379).

Due to the patient's death, the informed consent form was completed and signed by her parents.

\section{Discussion}

Fusarium is a common saprophytic fungus that is found in the soil, air, and water. Although Fusarium species were first isolated as phytopathogens (plant pathogens), they can cause fixed (onychomycosis and fungal keratitis) and systemic infections in humans, especially in immunocompromised patients $(4,5,9)$. These opportunistic fungi caused high morbidity and mortality rates among patients with malignancies, severe $\mathrm{T}$ cell immunodeficiency, and prolonged neutropenia. More than 120 Fusarium spp. has been identified in seven complexes: Fusarium solani, F. incarnatum-equiseti, F. oxysporum, F. dimerum, F. fujikuroi, F. sporotrichioides, and F. clamydosporum (10). Among these groups, Fusarium solani and Fusarium oxysporum complexes are responsible for about 65\% and 30\% of infections, respectively (11). The main entry ways of
Fusarium species are the airways, skin, and mucosal membranes $(12,13)$. Macrophages and granulocytes play significant roles in the immune system against Fusarium species. Both macrophages and granulocytes inhibit the growth of hypha, while macrophages inhibit the germination of spores (2). Among patients with neutropenia, the most repeated clinical signs of infection are fever, cutaneous lesions, and positive blood culture $(1,13)$ however, cutaneous lesions were not observed in the patient in the present study. Therapeutic approaches to fusariosis cases are rare and response to the treatment depends on the fungal species and the patient's immune status. In cancer patients, particularly those with prolonged neutropenia, fusariosis shows resistance to antifungal therapy. The mortality rate in patients with systemic fusariosis is apparently higher than those patients with localized infections specifically among patients with refractory neutropenia. Totally, amphotericin B lipid complex (ABLC) is suggested for the management of Fusarium infection, but the response is moderate among the neutropenic cancer patients (14). In the present case, the cause of death was not clear because the patient died 6 days after admission, probably was due to the invasive fungal infection or cancer recurrence. In 2004, Jensen et al. (15) reported 4 cases of fungemia due to Fusarium species in acute lymphoblastic leukaemia, chronic lymphatic leukaemia, acute myeloid leukaemia, and non-Hodgkin's lymphoma patients from Denmark. The etiological agents were Fusarium spp., F. verticillioides (syn. F. moniliforme), F. verticillioides, and F. solani. Except for one patient, all patients died despite antifungal therapy with intravenous amphotericin B (AmBisome). In 2010, Jossi et al. (7) presented a patient with acute lymphoblastic leukemia that revealed prolonged fungemia. The patient also had multiple sclerosis and invasive breast carcinoma. Despite antifungal therapy with fluconazole (200 $\mathrm{mg} \mathrm{PO} /$ day), voriconazole $(2 \times 300 \mathrm{mg}$ IV/day), and liposomal amphotericin B (5 $\mathrm{mg} / \mathrm{kg} /$ day IV), she died 16 days after infection. In 2016, Dabas et al. (5) reported two cases of systemic fusariosis in 17 and 65 years old patients with acute myeloid leukaemia and cutaneous $\mathrm{T}$ cell large anaplastic lymphoma erythroderma. Fusarium solani was isolated from two patients and both of them expired due to the severe sepsis and septic shock. In 2016, Narayanan and Nath (16) showed disseminated Fusarium fungemia in a 21-year-old woman with acute myeloid leukemia from India. Both legs of the patient had cellulitis and erythematous lesions with blackish center. In spite of antifungal therapy with amphotericin B and voriconazole, bronchopneumonia developed and the patient passed away. In 2017, Chiewchanvit et al. (17) presented a case of disseminated fusariosis in a 24-year-old fe- 

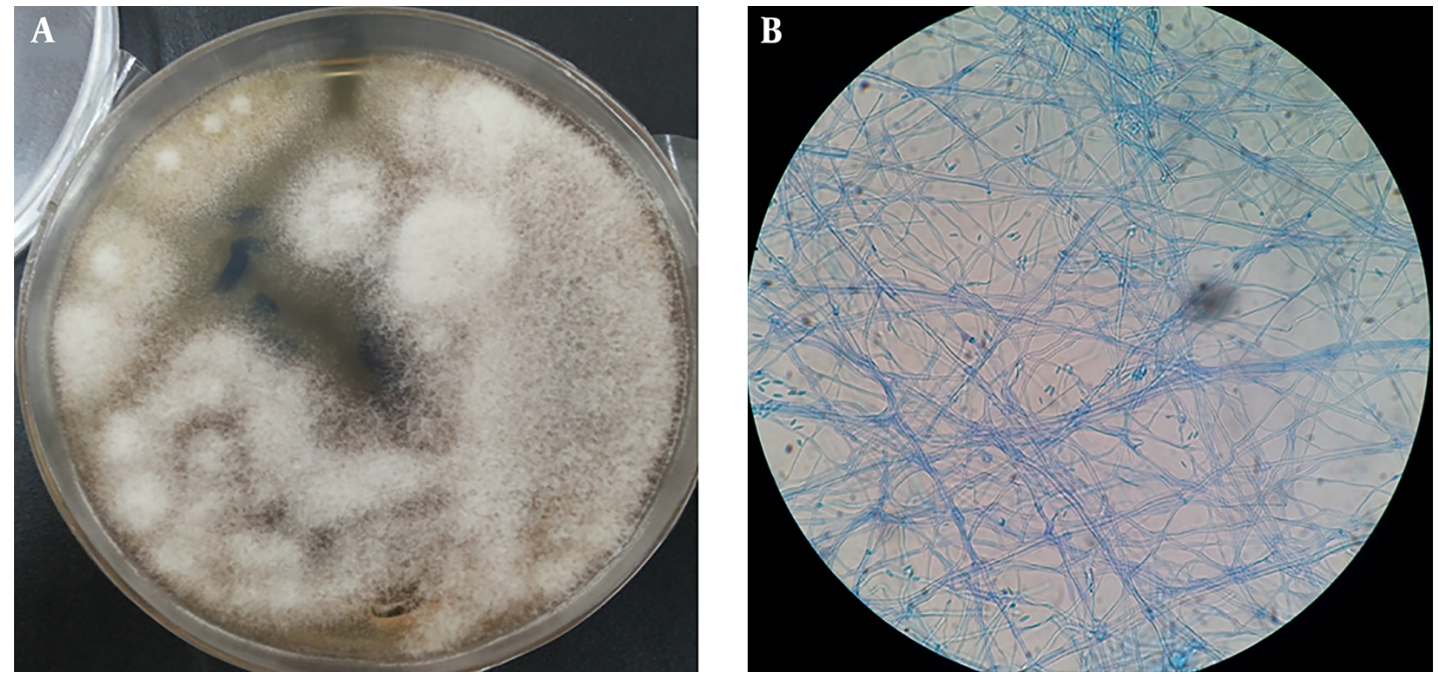

Figure 1. A, Fusarium solani on sabouraud dextrose agar (SDA) shows white and cottony hypha; B, microscopic examination with potassium hydroxide 10\% reveals typical fusiform microconidia among hyaline hyphae.

male with acute leukemia from Thailand. Erythematous papules were distributed on the extremities and trunk. The patient was treated with itraconazole $(100 \mathrm{mg} /$ day), intravenous amphotericin B (40 mg/day or $1 \mathrm{mg} / \mathrm{kg} /$ day), and oral voriconazole (400 mg/day). Fusarium keratoplasticum was identified as the etiological agent of fusariosis using multilocus sequencing typing (MLST) method. Carlesse et al. (18) reported an outbreak of catheter-related fungemia among children with cancer in Sao Paulo, Brazil. In their study, Fusarium oxysporum was the causative agents of all cases. They survived after prompt catheter removal and antifungal therapy with oral voriconazole ( $7 \mathrm{mg} / \mathrm{kg} /$ dose). In 2019, Yasu et al. (19) from Japan, presented a case of fungemia after cord blood transplantation in a 65-year-old man. The patient died due to the fungemia on day 28 after treatment with fosfluconazole (F-FLCZ) (100 mg/day) and L-AMB (50 mg/day) or ( $0.72 \mathrm{mg} / \mathrm{kg}$ ). Since, Fusarium solani is usually resistant to multiple antifungal agents, such as echinocandins and itraconazole (19), and the optimal dose of liposomal amphotericin B for Fusarium fungemia during neutropenia remains unclear, we highlight the use of wide spectrum antifungal agents, such as voriconazole and posaconazole as primary prophylaxis and combination therapies for vulnerable patients. Since identification of Fusarium spp. is controversy by phenotypic methods, we suggest a precise technique such as ITS-sequencing for identification of causative agent of fungemia. Since the mortality rate of fungemia due to the Fusarium spp. is high, we recommend primary prophylaxis with a broadspectrum antifungal agent for vulnerable patients partic- ularly those admitted to high-risk units such as oncology, hematology, and transplant units.

\section{Acknowledgments}

None decalred.

\section{Footnotes}

Authors' Contribution: Alireza Rajabzadeh detected fungal infection in the patient. Dariush Shokri, Shima Aboutalebian, Hamid Morovati, and Abdolrasoul Mohammadi, identified the fungus by phenotypic and molecular techniques under supervision of Rasoul Mohammadi. Alireza Rajabzadeh, and Reza Eshraghi Samani, managed the patient for cancer she involved. Rasoul Mohammadi provided the draft of manuscript and all authors read and confirmed the manuscript.

Conflict of Interests: The authors declare no conflicts of interest.

Funding/Support: All stages of fungal identification were supported by Isfahan University of Medical Sciences.

Informed Consent: Due to the patient's death, the informed consent form completed and signed by her parents.

\section{References}

1. Arnoni MV, Paula CR, Auler ME, Simoes CCN, Nakano S, Szeszs MW, et al. Infections caused by fusarium species in pediatric cancer patients 
and review of published literature. Mycopathologia.2018;183(6):941-9. doi: 10.1007/s11046-018-0257-6. [PubMed: 29564632].

2. Nucci M, Anaissie E. Fusarium infections in immunocompromised patients. Clin Microbiol Rev. 2007;20(4):695-704. doi: 10.1128/CMR.00014-07. [PubMed: 17934079]. [PubMed Central: PMC2176050].

3. Nelson PE, Dignani MC, Anaissie EJ. Taxonomy, biology, and clinical aspects of Fusarium species. Clin Microbiol Rev. 1994;7(4):479504. doi: 10.1128/cmr.7.4.479. [PubMed: 7834602]. [PubMed Central: PMC358338].

4. Muhammed M, Anagnostou T, Desalermos A, Kourkoumpetis TK Carneiro HA, Glavis-Bloom J, et al. Fusarium infection: report of 26 cases and review of 97 cases from the literature. Medicine (Baltimore). 2013;92(6):305-16. doi: 10.1097/MD.0000000000000008. [PubMed: 24145697]. [PubMed Central: PMC4553992].

5. Dabas Y, Bakhshi S, Xess I. Fatal cases of bloodstream infection by fusarium solani and review of published literature. Mycopathologia. 2016;181(3-4):291-6. doi: 10.1007/s11046-015-9960-8. [PubMed: 26541869].

6. Campo M, Lewis RE, Kontoyiannis DP. Invasive fusariosis in patients with hematologic malignancies at a cancer center: 1998-2009.J Infect. 2010;60(5):331-7. doi: 10.1016/j.jinf.2010.01.010. [PubMed: 20138081].

7. Jossi M, Ambrosioni J, Macedo-Vinas M, Garbino J. Invasive fusariosis with prolonged fungemia in a patient with acute lymphoblastic leukemia: Case report and review of the literature. Int J Infect Dis. 2010;14(4):e354-6. doi: 10.1016/j.ijid.2009.05.004. [PubMed: 19665409].

8. Kochl S, Niederstatter H, Parson W. DNA extraction and quantitation of forensic samples using the phenol-chloroform method and realtime PCR. Methods Mol Biol. 2005;297:13-30. doi: 10.1385/1-59259-8676:013. [PubMed: 15570097].

9. Konuma T, Takahashi S, Kiyuna T, Misawa Y, Suzuki M, Isobe M, et al. Fungemia due to Fusarium solani under low-dose liposomal amphotericin B in a patient after cord blood transplantation. J Infect Chemother. 2019;25(8):635-8. doi: 10.1016/j.jiac.2019.02.020. [PubMed: 30910505].

10. van Diepeningen AD, Brankovics B, Iltes J, van der Lee TA, Waalwijk C. Diagnosis of Fusarium infections: Approaches to identification by the clinical mycology laboratory. Curr Fungal Infect Rep. 2015;9(3):135-43. doi: 10.1007/s12281-015-0225-2. [PubMed: 26301000]. [PubMed Central: PMC4537702].
11. Guarro J. Fusariosis, a complex infection caused by a high diversity of fungal species refractory to treatment. Eur J Clin Microbiol Infect Dis. 2013;32(12):1491-500. doi:10.1007/s10096-013-1924-7. [PubMed 23934595].

12. Sautour M, Edel-Hermann V, Steinberg C, Sixt N, Laurent J, Dalle F, et al. Fusarium species recovered from the water distribution system of a French University Hospital. Int J Hyg Environ Health. 2012;215(3):28692. doi: 10.1016/j.ijheh.2011.11.003. [PubMed: 22177529].

13. Litvinov N, da Silva MT, van der Heijden IM, Graca MG, Marques de Oliveira L, Fu L, et al. An outbreak of invasive fusariosis in a children's cancer hospital. Clin Microbiol Infect. 2015;21(3):268 e1-7. doi: 10.1016/j.cmi.2014.09.004. [PubMed: 25658562].

14. Espinel-Ingroff A, Colombo AL, Cordoba S, Dufresne PJ, Fuller J, Ghannoum M, et al. International evaluation of MIC distributions and epidemiological cutoff value (ECV) Definitions for fusarium species identified by molecular methods for the CLSI broth $\mathrm{mi}$ crodilution method. Antimicrob Agents Chemother. 2016;60(2):107984. doi: 10.1128/AAC.02456-15. [PubMed: 26643334]. [PubMed Central: PMC4750715].

15. Jensen TG, Gahrn-Hansen B, Arendrup M, Bruun B. Fusarium fungaemia in immunocompromised patients. Clin Microbiol Infect 2004;10(6):499-501. doi: 10.111//j.1469-0691.2004.00859.x. [PubMed: 15191376].

16. Narayanan G, Nath SR. Disseminated Fusarium fungemia in a patient with acute myeloid leukemia. Mayo Clin Proc. 2016;91(4):542-3. doi 10.1016/j.mayocp.2015.11.006. [PubMed: 27046532].

17. Chiewchanvit S, Chongkae S, Mahanupab P, Nosanchuk JD, Pornsuwan S, Vanittanakom N, et al. Melanization of Fusarium keratoplasticum (F. solani species complex) during disseminated fusariosis in a patient with acute leukemia. Mycopathologia. 2017;182(9-10):879-85 doi: 10.1007/s11046-017-0156-2. [PubMed: 28616680].

18. Carlesse F, Amaral AC, Goncalves SS, Xafranski H, Lee MM, Zecchin V, et al. Outbreak of Fusarium oxysporum infections in children with cancer: an experience with 7 episodes of catheter-related fungemia. Antimicrob Resist Infect Control. 2017;6:93. doi: 10.1186/s13756-017-02473. [PubMed: 28912948]. [PubMed Central: PMC5588724].

19. Yasu T, Konuma T, Oiwa-Monna M, Kato S, Tanoue S, Isobe M, et al. Efficacy and safety of low-dose liposomal amphotericin B in adult patients undergoing unrelated cord blood transplantation. Antimicrob Agents Chemother. 2018;62(11). doi:10.1128/AAC.01205-18. [PubMed 30104271]. [PubMed Central: PMC6201127]. 